\title{
Embryo stage of development is not decisive for reproductive outcomes in frozen-thawed embryo transfer cycles
}

Bruno R de Carvalho ${ }^{1}$, Marina W Paes Barbosa ${ }^{1}$, Helena Bonesi ${ }^{1}$, David B Gomes Sobrinho ${ }^{1}$, Íris O Cabral ${ }^{1}$, Antônio C Paes Barbosa ${ }^{1}$, Adelino A Silva ${ }^{1}$, José R Iglesias ${ }^{1}$, Hitomi M Nakagawa ${ }^{1}$

${ }^{1}$ GENESIS - Center for Assistance in Human Reproduction, Brasília, DF, Brazil

\begin{abstract}
Objective: To evaluate if the outcomes of IVF/ICSI in frozen-thawed embryo transfer and fresh embryo transfer cycles differ in relation to cleavage and blastocyst stages.

Methods: Retrospective cohort study to compare IVF/ICSI outcomes between fresh embryo transfer and frozen-thawed embryo transfer cycles, according to the stage of embryo development. Analysis was carried out on 443 consecutive embryo transfer cycles performed between January 1st and December 31st, 2014. Women aged up to 38 and submitted to embryo transfer cycles with fresh $(n=309)$ or frozen-thawed $(n=134)$ embryos at a private center for assistance in human reproduction were considered for analysis. Results in each group were stratified according to the stage of embryo development: cleavage stage and blastocyst stage. Main outcome measures were implantation rate, clinical pregnancy rate, ongoing pregnancy rate and live birth rate per cycle.

Results: In the fresh embryo transfer group, for cleavage stage versus blastocyst stage, respectively, implantation rates were $22 \%$ and $47 \%(p=0.0005)$; clinical pregnancy rates were $34 \%$ and $64 \%(p=0.0057)$; the ongoing pregnancy rates were $30 \%$ and $61 \%(p=$ $0.0046)$ and live birth rates were $28 \%$ and $55 \%(p=$ 0.0148 ). There were no significant differences in the rates between cleavage and blastocyst stages in the frozenthawed group, neither between fresh and frozen-thawed cleavage embryo transfers nor between fresh and frozenthawed blastocyst transfers.

Conclusion: Our results confirm that blastocyst transfer is better than cleavage stage in fresh embryo transfer cycles. In frozen-thawed cycles, cleavage or blastocyst stages seem to offer similar reproductive outcomes.
\end{abstract}

Keywords: Embryo development, reproductive outcomes, frozen-thawed embryo transfer, blastocyst

\section{INTRODUCTION}

In Assisted Reproductive Techniques (ART), successful implantation depends not only on embryo quality, but also on endometrial receptivity (Evans et al., 2014). Traditionally, embryo transfers (ET) were performed on the cleavage stage, because the uterus was supposed to be the best environment for embryo survival (Laverge et al., 2001). On the other hand, understanding embryo metabolism and development of sequential culture media enabled embryo transfers on blastocyst stage (Gardner et al., 1998 a,b).

Theoretically, blastocysts present a higher implantation potential, since in vivo embryos reach the uterus at least on day 4 of fertilization (Gardner et al., 1996). There is also evidence that uterine contractility decreases at the time of blastocyst transfer, thus reducing the chance of embryo expulsion (Fanchin et al., 2001). Finally, after controlled ovarian stimulation, supraphysiological levels of estrogen may alter endometrial receptivity; blastocyst transfer would prevent premature exposure to an altered uterine environment (Gardner et al., 1998a,b; Valbuena et al., 2001; Fatemi \& Popovic-Todorovic, 2013; Roque et al., 2015).

This is also the reason for performing frozen-thawed ET. Since endometrial development is more precisely controlled in those cycles, it would be possible to prevent an embryo-endometrium asynchrony (Roque, 2015). However, clinical pregnancy rates (CPR) and live birth rates (LBR) do not seem to differ between frozen-thawed ET and fresh ET cycles, mainly if blastocysts are transferred.

The aim of this study was to evaluate if the outcomes of IVF/ICSI in frozen-thawed embryo transfer and fresh embryo transfer cycles differ according to the stage of embryo development.

\section{MATERIALS AND METHODS}

This was a retrospective cohort study to compare IVF/ ICSI outcomes between fresh ET and frozen-thawed ET, according to the stage of embryo development (cleavage versus blastocyst). The analysis was carried out on 443 consecutive ET cycles performed between January 1st and December 31st, 2014. The main outcomes were implantation rate, clinical pregnancy rate, ongoing pregnancy rate and live birth rate per cycle.

We studied the women aged up to 38 and submitted to ET cycles with fresh $(n=309)$ or frozen-thawed $(n=$ 134) embryos at a private center for assistance in human reproduction. Results in each group were stratified according to the stage of embryo development: cleavage stage (from day 2 to day 4 ) and blastocyst stage (days 5 and 6). Table 1 presents patients' characteristics per treatment group. The evaluated outcomes were biochemical pregnancy, clinical pregnancy, ongoing pregnancy and live birth rates.

The Institutional Clinical Committee approved the study. Patients gave written consent for assisted reproduction technology treatment, and an oral consent was obtained for confidential data use for research purposes. A specific written informed consent was not considered necessary for this study, since the data was obtained exclusively from patient files, respecting anonymity.

The statistical analysis was performed using GraphPad Prism software, version 5.00 (GraphPad Software, Inc, 2007). Fisher's exact test was used to compare rates between groups. The level of significance was set at $p<$ 0.05 in all analyses.

\section{RESULTS}

In the fresh ET group, for cleavage stage versus blastocyst stage, the implantation rates were $22 \%$ and $47 \%(p=0.0005)$, respectively; clinical pregnancy rates were $34 \%$ and $64 \%(p=0.0057)$, ongoing pregnancy rates 


\begin{tabular}{|l|c|c|c|}
\hline Table 1. Patients' characteristics per treatment group. & Blastocyst & $\boldsymbol{p}^{\text {value }}$ \\
\hline Outcomes & Cleavage & & \\
\hline Fresh ET & & $33.03 \pm 2.26$ & $\mathrm{NS}$ \\
\hline Age, years & $33.9 \pm 3.25$ & $1.94 \pm 0.35$ & $\mathrm{NS}$ \\
\hline Embryos transferred, $\mathrm{n}$ & $2.04 \pm 0.52$ & & $\mathrm{NS}$ \\
\hline Frozen-thawed ET & & $31.64 \pm 4.02$ & 0.0486 \\
\hline Age, years & $32.9 \pm 3.96$ & $1.85 \pm 0.42$ & \\
\hline Embryos transferred, $\mathrm{n}$ & $2.05 \pm 0.49$ & & \\
\hline
\end{tabular}

The data is expressed as means \pm standard deviations

ET = embryo transfer; NS = not significant

aStatistical analysis performed by unpaired $t$-test or Mann-Whitney test or Fisher's exact test

were $30 \%$ and $61 \%(p=0.0046)$ and live birth rates were $28 \%$ and $55 \%(p=0.0148)$. There were no significant differences in rates between cleavage and blastocyst stages in the frozen-thawed ET group (Table 2).

\section{DISCUSSION}

Our results confirm that blastocyst transfer is better than cleavage stage ET in fresh cycles. Our findings are in agreement with both systematic reviews assessing this comparison, which favored blastocyst culture for improving live birth rates (Papanikolaou et al., 2008; Glujovsky et al., 2012). The most likely explanation is that blastocysts may be able to overcome the negative impact by controlled ovarian stimulation on endometrial receptivity (Gardner et al., 1998a,b; Valbuena et al., 2001). Besides, it seems that there is a natural selection through embryonic development, which does not allow most of the chromosomally abnormal embryos to reach higher developmental stages, and ultimately to implant (Gardner et al., 1998 a,b; Papanikolaou et al., 2008).

On the other hand, in frozen-thawed ET cycles, cleavage or blastocyst stages seemed to offer similar reproductive outcomes. This was probably because the non-stimulated endometrium (or minimally stimulated) in those cycles may be highly effective in equalizing the results for embryos at any stage of development.

There are several factors that explain why supraphysiological steroid levels impair embryo implantation. First of all, controlled ovarian stimulation (COS) leads to advancement of pinopodes appearance and histological features linked to implantation, resulting in an embryo-endometrium asynchrony (Mirkin et al., 2004). There is also a progesterone receptor down-regulation in glandular and stromal cells, leading to a desynchrony, where glandular and stromal maturation do not match the same day (Valbuena et al., 2001; Mirkin et al., 2004).

Another point is the evidence that COS causes deregulation of more than 200 genes related to

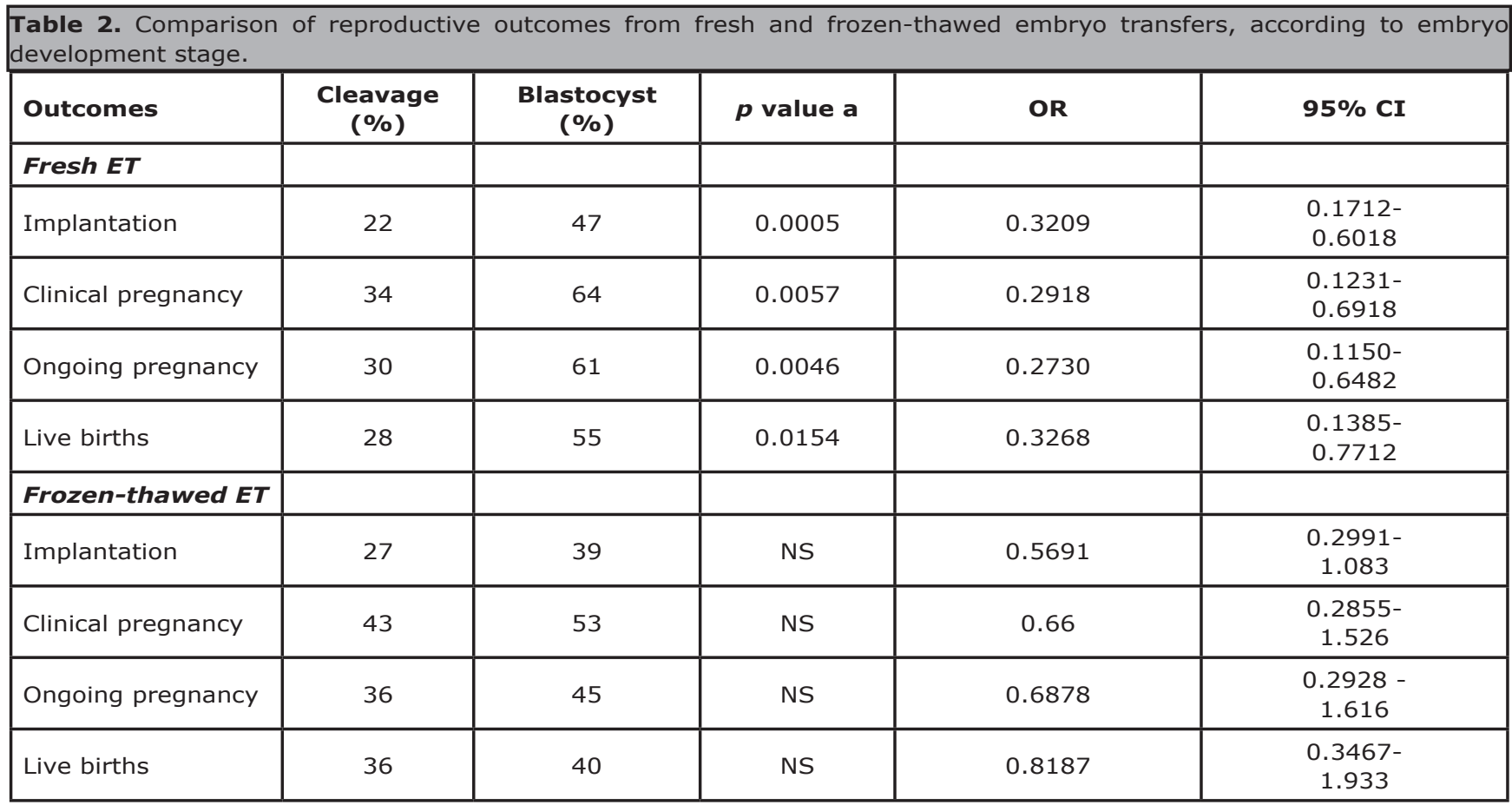

Outcomes presented: Implantation: the ratio of the number of fetal hearts to the number of embryos transferred; Clinical pregnancy: an ultrasound confirmed fetal heart after the 6th gestational week; Ongoing pregnancy: pregnancy continued after the 14th gestational weekET = embryo transfer; NS = not significant; OR = odds ratio; $\mathrm{CI}=$ confidence interval aStatistical analysis performed by unpaired $t$-test or Mann-Whitney test or Fisher's exact test 
implantation, in comparison to natural cycles. The altered gene expression profile leads to an aberrant endometrium, which probably impairs the ideal intrauterine microenvironment for embryo implantation (Horcajadas et al., 2005; Labarta et al., 2011).

Moreover, elevated estrogen concentration may increase uterine contractions. This effect is more important for cleavage ET, since the contractility decreases progressively, reaching a nearly quiescent status at the time of the blastocyst transfer (Fanchin et al., 2001).

Based on the obtained data, we were not able to find significant differences in rates between fresh and frozenthawed cleavage ET or between fresh and frozen-thawed blastocyst ET. Previous studies have demonstrated similar results for cleavage stage embryos (Bdolah et al., 2015), but conflicting results have been published regarding blastocyst transfers (Feng et al., 2012; Doherty et al., 2014; Özgür et al., 2015; Gomaa et al., 2016).

The existence of biases related to the retrospective design of our study cannot be excluded and our results must be considered with caution. Statistical analysis demonstrated a small but significant difference in the numbers of embryos transferred between frozen-thawed cleavage and blastocyst transfers; thus, implantation rates may be misleading (Griesinger, 2016). Also, our study did not explore the number or even the existence of top quality embryos at the freezing, thawing and transfer times, which are supposed to be factors that may negatively influence results. There is recent evidence that transferring one top quality embryo may be more important to improve the chance of a live birth than endometrial function in frozenthawed cycles (Veleva et al., 2013), and it may be related to a higher PR (Salumets et al., 2006).

Besides, our study design limited exploration of other factors that may influence reproductive outcomes, such as body mass index, etiopathogenic diagnosis of infertility, primary versus and secondary infertility, pregnancy in a previous fresh cycle, type of luteal support, precocious progesterone elevation.

In conclusion, our study suggests that blastocysts are the best option for fresh ET. However, in frozen-thawed ET, no significant differences in reproductive outcomes were found between cleavage and blastocyst stages.

\section{CONFLICT OF INTERESTS}

The authors declare there were no potential conflicts of interests of financial or other nature, which may be deemed to influence the objectivity of the manuscript. Also, there was no funding received for this study from any organization.

\section{Corresponding authors:}

Hitomi Miura Nakagawa

GENESIS - Center for Assistance in Human Reproduction Brasília, DF, Brazil.

E-mail: naka.miuramd@gmail.com

Bruno Ramalho de Carvalho

E-mail: brunoramalho@hotmail.com

\section{REFERENCES}

Bdolah Y, Zemet R, Aizenman E, Lossos, Abram TB, Shufaro Y. Frozen-Thawed Embryo Transfer Success Rate is Affected by Age and Ovarian Response at Oocyte Aspiration Regardless of Blastomere Survival Rate. JBRA Assist Reprod. 2015;19:210-5. PMID: 27203194 DOI: https://doi.org/10.5935/1518-0557.20150041
Doherty LF, Martin JR, Kayisli U, Sakkas D, Patrizio P. Fresh transfer outcome predicts the success of a subsequent frozen transfer utilizing blastocysts of the same cohort. Reprod Biomed Online. 2014;28:204-8. PMID: 24365019 DOI: http://dx.doi.org/10.1016/j.rbmo.2013.09.030

Evans J, Hannan NJ, Edgell TA, Vollenhoven BJ, Lutjen PJ, Osianlis T, Salamonsen LA, Rombauts LJ. Fresh versus frozen embryo transfer: backing clinical decisions with scientific and clinical evidence. Hum Reprod Update. 2014;20:808-21. PMID: 24916455 DOI: http://dx.doi.org/10.1093/humupd/dmu027

Fanchin R, Ayoubi JM, Righini C, Olivennes F, Schönauer LM, Frydman R. Uterine contractility decreases at the time of blastocyst transfers. Hum Reprod. 2001;16:1115-9. PMID: 11387279 DOI: http://dx.doi.org/10.1093/humrep/16.6.1115

Fatemi HM, Popovic-Todorovic B. Implantation in assisted reproduction: a look at endometrial receptivity. Reprod Biomed Online. 2013;27:530-8. PMID: 23933035 DOI: http://dx.doi.org/10.1016/j.rbmo.2013.05.018

Feng G, Zhang B, Zhou H, Shu J, Gan X, Wu F, Deng X. Comparable clinical outcomes and live births after single vitrified-warmed and fresh blastocyst transfer. Reprod Biomed Online. 2012;25:466-73. PMID: 22995746 DOI: http://dx.doi.org/10.1016/j.rbmo.2012.07.008

Gardner DK, Lane M, Calderon I, Leeton J. Environment of the preimplantation human embryo in vivo: metabolite analysis of oviduct and uterine fluids and metabolism of cumulus cells. Fertil Steril. 1996;65:349-53. PMID: 8566260 DOI: http://dx.doi.org/10.1016/S0015-0282(16)58097-2

Gardner DK, Schoolcraft WB, Wagley L, Schlenker T, Stevens J, Hesla J. A prospective randomized trial of blastocyst culture and transfer in in-vitro fertilization. Hum Reprod. 1998a;13:3434-40. PMID: 9886530 DOI: http://dx.doi.org/10.1093/humrep/13.12.3434

Gardner DK, Vella P, Lane M, Wagley L, Schlenker T, Schoolcraft WB. Culture and transfer of human blastocysts increases implantation rates and reduces the need for multiple embryo transfers. Fertil Steril. 1998b;69:84-8. PMID: 9457939 DOI: http://dx.doi.org/10.1016/S0015-0282(97)00438-X

Glujovsky D, Blake D, Farquhar C, Bardach A. Cleavage stage versus blastocyst stage embryo transfer in assisted reproductive technology. Cochrane Database Syst Rev. 2012;(7):CD002118. PMID: 22786480 DOI: https://doi.org/10.1002/14651858.CD002118.pub4

Gomaa H, Baydoun R, Sachak S, Lapana I, Soliman S. Elective single embryo transfer: Is frozen better than fresh? JBRA Assist Reprod. 2016;20:3-7. PMID: 27203298 DOI: http://dx.doi.org/10.5935/1518-0557.20160002

Griesinger G. Beware of the 'implantation rate'! Why the outcome parameter 'implantation rate' should be abandoned from infertility research. Hum Reprod. 2016;31:249-51. PMID: 26724801 DOI: https://doi.org/10.1093/humrep/dev322 
Horcajadas JA, Riesewijk A, Polman J, van Os R, Pellicer A, Mosselman S, Simón C. Effect of controlled ovarian hyperstimulation in IVF on endometrial gene expression profiles. Mol Hum Reprod. 2005;11:195-205. PMID: 15695772 DOI: http://dx.doi.org/10.1093/molehr/gah150

Labarta E, Martínez-Conejero JA, Alamá P, Horcajadas JA, Pellicer A, Simón C, Bosch E. Endometrial receptivity is affected in women with high circulating progesterone levels at the end of the follicular phase: a functional genomics analysis. Hum Reprod. 2011;26:1813-25. PMID: 21540246 DOI: http://dx.doi.org/10.1093/humrep/der126

Laverge $H$, De Sutter $P$, Van der Elst J, Dhont $M$. A prospective, randomized study comparing day 2 and day 3 embryo transfer in human IVF. Hum Reprod. 2001;16:476-80. PMID: 11228214 DOI: http://dx.doi.org/10.1093/humrep/16.3.476

Mirkin S, Nikas G, Hsiu JG, Díaz J, Oehninger S. Gene expression profiles and structural/functional features of the peri-implantation endometrium in natural and gonadotropin-stimulated cycles. J Clin Endocrinol Metab. 2004;89:5742-52. PMID: 15531538 DOI: http://dx.doi.org/10.1210/jc.2004-0605

Özgür K, Berkkanoglu M, Bulut H, Isikli A, Coetzee K. Higher clinical pregnancy rates from frozen-thawed blastocyst transfers compared to fresh blastocyst transfers: a retrospective matched-cohort study. J Assist Reprod Genet. 2015;32:1483-90. PMID: 26400506 DOI: http://dx.doi.org/10.1007/s10815-015-0576-1
Papanikolaou EG, Kolibianakis EM, Tournaye H, Venetis CA, Fatemi H, Tarlatzis B, Devroey P. Live birth rates after transfer of equal number of blastocysts or cleavage-stage embryos in IVF. A systematic review and meta-analysis. Hum Reprod. 2008;23:91-9. PMID: 17965420 DOI: http://dx.doi.org/10.1093/humrep/dem339

Roque M. Freeze-all policy: is it time for that? J Assist Reprod Genet. 2015;32:171-6. PMID: 25428436 DOI: http://dx.doi.org/10.1007/s10815-014-0391-0

Roque M, Valle M, Guimarães F, Sampaio M, Geber S. Freeze-all policy: fresh vs. frozen-thawed embryo transfer. Fertil Steril 2015;103:1190-3. PMID: 25747130 DOI: http://dx.doi.org/10.1016/j.fertnstert.2015.01.045

Salumets A, Suikkari AM, Mäkinen S, Karro H, Roos A, Tuuri T. Frozen embryo transfers: implications of clinical and embryological factors on the pregnancy outcome. Hum Reprod. 2006;21:2368-74. PMID: 16684837 DOI: http://dx.doi.org/10.1093/humrep/del151

Valbuena D, Martin J, de Pablo JL, Remohí J, Pellicer A, Simón C. Increasing levels of estradiol are deleterious to embryonic implantation because they directly affect the embryo. Fertil Steril. 2001;76:962-8. PMID: 11704118 DOI: http://dx.doi.org/10.1016/S0015-0282(01)02018-0

Veleva Z, Orava M, Nuojua-Huttunen S, Tapanainen JS, Martikainen $\mathrm{H}$. Factors affecting the outcome of frozen-thawed embryo transfer. Hum Reprod. 2013;28:2425-31. PMID: 23756705 DOI: http://dx.doi.org/10.1093/humrep/det251 